\title{
Exposições ambientais e leucemias na infância no Brasil: uma análise exploratória de sua associação
}

\author{
Jeniffer Dantas Ferreira* \\ Arnaldo Cézar Couto** \\ Luciana Correia Alves ${ }^{\star \star *}$ \\ Maria do Socorro Pombo de Oliveira ${ }^{\star \star \star *}$ \\ Sergio Koifman ${ }^{\star \star \star \star \star *}$ \\ Brazilian Collaborative Study Group of Infant Acute Leukemia ${ }^{\star \star \star \star \star \star}$
}

\begin{abstract}
O presente estudo objetiva explorar as relações inaparentes que diversos fatores relativos às exposições ambientais e características individuais existentes em nosso meio possam ter no processo de desenvolvimento da leucemia na infância. A partir de um banco de dados clínicos e epidemiológicos obtido com estudo caso-controle de base hospitalar sobre fatores de risco para leucemias na infância, foi realizada análise multivariada exploratória por meio do emprego de análise de componentes principais e análise fatorial. Esta investigação é parte de um estudo multicêntrico nacional, que incluiu 292 casos de leucemias em crianças com idade entre 0 e 12 anos e 541 controles da mesma faixa etária, hospitalizados por causas não neoplásicas em hospitais gerais próximos aos centros de origem dos casos. As informações de exposições ambientais selecionadas foram obtidas por meio de entrevistas realizadas com mães de casos e controles, por meio de questionário padronizado. O modelo com maior poder explicativo da variância observada nos dados analisados foi da ordem de $52 \%$, apresentando três fatores considerados mais adequados na predição da leucemogênese na infância, cada um incluindo variáveis com cargas fatoriais maiores que 0,6: fator "condições relacionadas a exposições químicas na gestação", o qual explicou $20 \%$ da variância final e incluiu as variáveis exposição a pesticidas, exposição a solventes e exposição a tintas na gravidez; fator "hábitos de vida durante a gestação", tendo explicado 17\% da variância e incluindo exposição a tinturas de cabelo e cosméticos para alisamento; e fator "consumo de serviços de saúde durante a gestação", explicando $15 \%$ da variância e incluindo as variáveis tipo de parto (cesariana ou parto normal) e uso de radiografia na gestação. Na análise de regressão logística, foi encontrada associação estatisticamente significativa entre o desenvolvimento de leucemias na infância e antecedentes de exposição química materna durante a gestação $(O R=1,36 ; 95 \% / C=1,16-1,59)$ e consumo de serviços
\end{abstract}

\footnotetext{
* Enfermeira e doutoranda no Programa de Pós-Graduação em Saúde Pública e Meio Ambiente, Escola Nacional de Saúde Pública Sergio Arouca - ENSP, Fiocruz/RJ.

**Biólogo e doutorando no Programa de Pós-Graduação em Saúde Pública e Meio Ambiente, Escola Nacional de Saúde Pública Sergio Arouca - ENSP, Fiocruz/RJ.

${ }^{* * *}$ Fisioterapeuta e pesquisadora visitante no Programa de Pós-Graduação em Saúde Pública e Meio Ambiente, Escola Nacional de Saúde Pública Sergio Arouca - ENSP, Fiocruz/RJ.

${ }_{* \star * *}$ Médica e pesquisadora na Divisão de Medicina Experimental, Centro de Pesquisa, Instituto Nacional de Câncer/RJ.

***** Médico e coordenador do Programa de Pós-Graduação em Saúde Pública e Meio Ambiente, Escola Nacional de Saúde Pública Sergio Arouca - ENSP, Fiocruz/RJ.

${ }^{\star * \star \star \star *}$ Membros do Brazilian Collaborative Study Group of Infant Acute Leukemia, listados ao final.
} 
de saúde durante a gestação $(O R=1,27 ; 95 \% / C=1,08-1,49)$. Os resultados encontrados são sugestivos quanto à contribuição conjunta das exposições ambientais, e não apenas individualizadas, no desenvolvimento das leucemias na infância, sendo apoiados pelas evidências na literatura de que o processo de carcinogênese, em geral, e o da leucemogênese, em particular, resultem de efeitos de múltiplas mutações relacionadas a exposições ambientais conjuntas.

Palavras-chave: Leucemia. Infância. Exposições ambientais. Análise fatorial. Regressão logística.

\section{Introdução}

As leucemias da infância (crianças com idade inferior ou igual a cinco anos) constituem um grupo heterogêneo de doenças, no que diz respeito tanto às expressões imunofenotípicas e morfológicas, quanto a seus mecanismos etiológicos. Apesar de tais doenças serem raras, a incidência da leucemia aguda corresponde a cerca de $30 \%$ dos diagnósticos de câncer pediátrico, sendo mais comum em menores de 15 anos (LINET et al., 1999; BRAGA et al., 2002; PUI et al., 2003). No que tange a taxa de mortalidade, observa-se um decréscimo desde a década de 1970 em países desenvolvidos (LA VECCHIA et al., 1998).

A leucemia aguda apresenta-se em duas categorias biologicamente distintas: linfoide (LLA) e mieloide (LMA). Os subgrupos de LLA são os mais frequentes, correspondendo a $75 \%$ dos casos leucêmicos pediátricos, com incidência mais elevada na faixa entre 2 e 5 anos (BHATIA et al., 1999; BELSON et al., 2007), enquanto a LMA não apresenta nenhuma associação com esta faixa etária.

A doença consiste em uma desordem do sistema hematopoiético decorrente de uma ou mais mutações que resultam num processo multifatorial, por meio da combinação de fatores genéticos, ambientais e de susceptibilidade individual (POMBO-DE-OLIVEIRA et al., 1986; PERERA, 1996; HUNGER et al., 1998; BHATIA et al., 1999; LABUDA et al., 2002; BELSON et al., 2007; HAMERSCHLAK, 2008).

Além do acúmulo de alterações na estrutura ou expressão de certos genes vitais - devido a mutações, translocações, perdas cromossômicas e recombinações somáticas -, outros fatores podem influenciar a susceptibilidade individual, tais como genes de alto e baixo risco, grupo étnico, idade, sexo, fatores nutricionais e de saúde (PERERA, 1996).

Um estudo publicado por Kinlen (1995) levantava a hipótese de que os deslocamentos populacionais ocorridos na Escócia com a imigração de trabalhadores de outros países, introduzindo a circulação de novos agentes biológicos, poderiam estar associados com a ascensão da taxa de incidência da leucemia infantil. As observações do estudo fomentaram a proposta de que alguns subtipos leucêmicos possam resultar de infecções comuns.

Greaves (1999), por sua vez, adaptou a teoria de "dois golpes" de Knudson, propondo que mutações aleatórias ocorreriam ainda na fase fetal e que a ausência de agentes infecciosos na primeira infância e subsequentes infecções tardias causariam desregulação na resposta imune, constituindo um segundo evento, proliferando um clone leucêmico latente.

Assim, diversas exposições durante o período gestacional poderiam desencadear mutações intrauterinas, acrescidas de uma exposição pós-natal (segundo golpe), resultando nas leucemias na infância.

Desde então, fatores têm sido apontados como responsáveis pelo processo de leucemogênese. Dentre estes, destacam-se a exposição a substâncias químicas (IARC, 1987; POMBO-DE-OLIVEIRA; KOIFMAN, 2006), radiações ionizantes e não-ionizantes (BELSON et al., 2007; SHU et al., 2002; WAKERFORD et al., 2003; WERTHEIMER; LEEPER, 1979; INFANTE-RIVARD et al., 2003), infecções (KINLEN, 1995; MCNALLY et al., 2004; DICKINSON, 2005) e outros 
agentes que possam estar associados aos polimorfismos e fusões gênicas.

No Brasil, de acordo com dados do Registro de Câncer de Base Populacional (RCBP), as taxas de incidência de leucemia na infância diferem entre as faixas etárias, os Estados brasileiros e por sexo, sendo maior no sexo masculino em quase todas as localidades. As taxas médias de incidência ajustadas por idade variaram, no período de 1996 a 2000, entre 0,33/100.000 em João Pessoa e 2,13/100.000 em São Paulo, para o sexo feminino, e 1,11/100.00 em João Pessoa e 3,19/100.000 em Natal, para o masculino (REIS et al., 2007).

Entretanto, são poucos os estudos no Brasil que focalizam a natureza dos fatores etiológicos envolvidos na gênese das leucemias pediátricas. $O$ presente trabalho foi realizado com o objetivo de explorar as relações inaparentes que diversas condições relacionadas a exposições ambientais e características individuais existentes em nosso meio possam ter no processo de desenvolvimento das leucemias na infância na população brasileira.

\section{Metodologia}

\section{Desenho e população de estudo}

Esta investigação faz parte de um estudo multicêntrico intitulado "Estudo Multi-institucional das Leucemias Infantis: Contribuição dos Marcadores Imuno-moleculares na Distinção de Diferentes Fatores Etiopatogênicos", que tem como foco investigar os mecanismos patogênicos das leucemias agudas da infância no Brasil.

Trata-se de um estudo caso-controle de base hospitalar, no qual os casos de leucemia na infância foram pareados por frequência de idade e local de residência com os controles. Entre 1991 e 2007, 695 casos foram diagnosticados como leucemias agudas, confirmados pelos métodos morfológicos, imunofenotípicos e citogenético-molecular. Destes, as investigações foram realizadas com leucemias em lactentes (idade inferior a 24 meses) e leucemias em crianças na faixa etária entre 2 e 12 anos. Os dados analisados foram obtidos por entrevistas realizadas com 292 mães de casos de leucemia e 541 mães de controles, constituindo a população analisada neste estudo $(n=833)$.

Os 541 controles aqui analisados foram selecionados nos hospitais gerais próximos aos centros de origem dos casos (Mapa 1), pertencentes ao mesmo estrato de idade e em tratamento para outras doenças não malignas. As crianças selecionadas como controles apresentaram antecedentes de hospitalização por diversas patologias, com a finalidade de reduzir-se a ocorrência de possível viés de seleção, e todas apresentando quadro de gravidade, com o intuito de reduzir a possível introdução de viés de memória relativa às informações coletadas, referentes aos fatores de riscos em leucemias pediátricas.

Os diagnósticos clínicos dos controles incluídos foram: diarreia, desnutrição, gastroenterites, asma, bronquite, pneumonia, arritmias, cardiopatias congênitas, hemangiomas, anemias ferroprivas e hemolíticas, crises convulsivas, toxoplasmose e calazar. Foram excluídos controles com diagnóstico de má formação congênita, portadores de Sida (Síndrome da Imunodeficiência Adquirida) e tumores malignos ou benignos devido à possível influência ambiental na formação de quaisquer tumores.

Os critérios de exclusão para a participação do estudo foram crianças com pais adotivos, mães biológicas não localizadas ou crianças cujas mães biológicas recusaram o convite à participação do estudo. Crianças portadoras de mielodisplasias ou Síndrome de Down também foram excluídas do estudo.

\section{Coleta de dados}

A coleta dos dados ocorreu por meio de entrevistas (face-a-face) com as mães de casos e controles, mediante o emprego de questionário padronizado e especificamente desenhado para obter informações sobre exposições ambientais potencialmente associadas ao processo de leucemogênese. Foram coletadas informações sobre o perfil socioeconômico da família, a história ocupacional da mãe e a do pai, os hábitos 
MAPA 1

Distribuição dos centros diagnósticos participantes do estudo

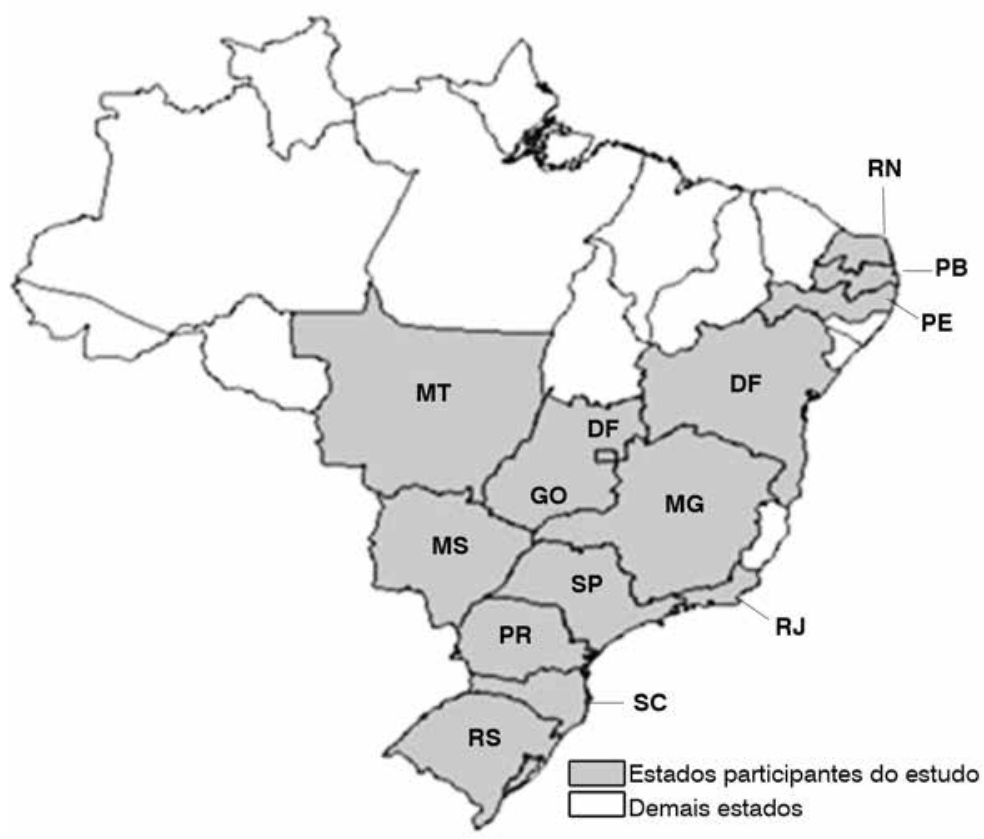

de vida e de saúde de ambos, exposições durante a gestação, tais como uso de medicamentos e substâncias psicoativas, e exposição materna a pesticidas, herbicidas e inseticidas, sendo coletadas informações sobre os respectivos produtos da gestação ao nascimento.

\section{Variáveis do estudo}

As características sociodemográficas familiares foram obtidas a partir dos relatos maternos quanto às variáveis sexo, cor da pele (brancos e não brancos), idade da criança no momento da entrevista ou no diagnóstico, peso ao nascer (g), comprimento ao nascimento $(\mathrm{cm})$, idades materna e paterna e renda familiar (salário mínimo de referência no período de confecção do questionário). Os dados pertinentes ao consumo dos serviços de saúde materna foram coletados por meio das variáveis tipo de parto (normal ou cesária), tipo de anestesia (raquidiana, peridural ou geral) e realização de transfusão sanguínea, ultrassonografia e radiografia.

As características relacionadas ao histórico gestacional foram obtidas a partir dos dados sobre acompanhamento pré-natal, ocorrência de abortos prévios (variável dicotômica), partos gemelares (variável dicotômica), aleitamento materno (uso ou não desta prática e período em meses), uso de fármacos durante a gestação (usou ou não fez uso) e diagnósticos, na gestação, de anemia, hipertensão arterial, infecção urinária ou ginecológica (presença ou ausência).

Os hábitos de vida dos pais foram identificados pelas seguintes variáveis: consumo de drogas ilícitas durante a gestação (uso ou não); consumo materno de fumo e álcool na gestação (uso ou não no período pré-concepcional, $1^{\circ}, 2^{\circ}$ e $3^{\circ} \circ$ trimestre da gestação e durante a lactação, quantidade e frequência de exposição); e tintura de cabelo e/ou alisamento materno três meses antes e durante a gestação e/ou amamentação (uso ou não) e tintura de cabelo e/ou alisamento 
paterno três meses antes da gestação (uso ou não).

Foi considerado exposição ambiental ou ocupacional o contato materno - ao menos uma vez nos três meses antes e durante a gestação e/ou amamentação - a: pesticidas, herbicidas e/ou inseticidas; poeiras (poeiras metálicas, areia, cimento, concreto, madeira, carvão, fuligem, algodão, lã e/ou fibra de vidro); motores (motores ou máquinas movidos a gasolina, diesel, álcool, combustíveis, coque, carvão, plástico, madeira e/ou outros); óleos (lubrificantes, fluido de corte, óleo mineral e/ou outros); solventes (desengraxantes, aguarraz, tricloroetileno, percloroetileno e/ou outros); tintas; e outras substâncias químicas (creosoto, asfalto ou betume, ácido clorídrico ou sulfúrico, colas e/ou outros).

Os antecedentes de história familiar de câncer (qualquer tipo de câncer), o uso de chupetas pela criança (uso ou não) e o relato sobre a presença de fumantes na residência (ao menos um morador tabagista durante a gestação) foram igualmente determinados.

\section{Análise estatística}

Para o estudo das relações inaparentes associadas à ocorrência de leucemia na infância, foram utilizadas a análise de componentes principais e a análise fatorial exploratória. O objetivo da análise fatorial é definir o relacionamento entre as variáveis de modo simples, utilizando menor número de variáveis do que o número original, reduzindo a dimensionalidade de um conjunto de variáveis por meio da utilização de suas intercorrelações. Em outras palavras, procura-se observar um conjunto de variáveis não observáveis a partir de um conjunto de variáveis observáveis (MINGOTI, 2005; MARCHIONI et al., 2005). Neste estudo, o conjunto de informações obtidas foi reduzido para 62 variáveis, que foram empregadas na análise.

Os testes Kaiser-Meyer-Olkin (KMO) e Bartlett foram usados para avaliar a adequação do emprego da análise fatorial, considerando-se o conjunto de dados analisados (HAIR et al., 1998). O KMO é considerado adequado quando for supe- rior a 0,5 e o Bartlett com a rejeição da hipótese nula.

A carga fatorial considerada foi acima de 0,3 para contribuir significativamente para o fator, após a rotação ortogonal pelo método varimax. Com o intuito de verificar a associação de cada fator com a presença de leucemia infantil, foi utilizado o modelo de regressão logística não condicional, sendo obtidas as razões de chance (odds ratios, OR) com os respectivos intervalos de confiança de 95\% (HOSMER; LEMESHOW, 1989).

As análises estatísticas foram realizadas pelo Statistical Package for Social Science (SPSS) versão 17.0.

\section{Resultados}

As análises de componentes principais e fatorial foram realizadas no conjunto de casos e controles, observando-se teste de KMO de 0,75 e o teste de esfericidade de Bartlett estatisticamente significativo ( $p$-valor $<0,0001)$. A análise de componentes principais revelou que $49 \%$ do total da variância do modelo foi explicado por quatro fatores, sendo estes identificados a partir dos critérios de Kaiser e Eigeinvalue (Tabela 1).

A rotação varimax apresentou cargas fatoriais mais elevadas no primeiro fator constituído pelas variáveis tabagismo materno durante a gestação, período da gestação onde ocorreu o consumo de fumo (1으, 2 은 e/ou 3o trimestre da gestação) e residir com fumantes durante a gestação. Devido à natureza das variáveis compondo este fator, o qual explicou $21 \%$ da variância dos dados, ele foi denominado condições associadas ao hábito de fumar na gravidez.

O segundo fator, responsável por $11,4 \%$ da variância, foi composto pelas variáveis exposição materna a: pesticidas, inseticidas e herbicidas; poeiras (metálicas, areia, cimento, concreto, madeira, carvão, fuligem, algodão, lã e/ou fibra de vidro); óleos (lubrificantes, fluido de corte, mineral e/ou outros); motores (ou máquinas movidas a gasolina, diesel, álcool, combustíveis, coque, carvão, plástico, madeira e/ou outros); solventes (e desengraxantes, aguarraz, tricloroetileno, percloroetileno e/ou outros); tintas; e outras 
TABELA 1

Carga fatorial obtida pela rotação varimax

\begin{tabular}{|c|c|c|c|c|}
\hline Variáveis & $\begin{array}{c}\text { Condições } \\
\text { associadas ao } \\
\text { hábito de fumar } \\
\text { na gravidez }\end{array}$ & $\begin{array}{c}\text { Exposição } \\
\text { ambiental ou } \\
\text { ocupacional }\end{array}$ & $\begin{array}{l}\text { Assistência } \\
\text { obstétrica }\end{array}$ & $\begin{array}{c}\text { Hábitos } \\
\text { de vida e } \\
\text { acesso a } \\
\text { serviços de } \\
\text { saúde }\end{array}$ \\
\hline Fumo durante a gestação & 0,966 & $-0,049$ & 0,006 & 0,038 \\
\hline Consumo de fumo no 10 trimestre & 0,981 & $-0,059$ & 0,017 & 0,040 \\
\hline Consumo de fumo no $2 \mathrm{o}$ trimestre & 0,982 & $-0,050$ & 0,006 & 0,028 \\
\hline Consumo de fumo no 3 o trimestre & 0,974 & $-0,044$ & $-0,014$ & 0,031 \\
\hline Morar com fumantes durante a gestação & 0,360 & 0,075 & $-0,136$ & 0,248 \\
\hline Contato materno durante a gestação com pesticidas & $-0,053$ & 0,384 & $-0,096$ & 0,109 \\
\hline Contato materno durante a gestação com poeiras & $-0,034$ & 0,574 & $-0,013$ & 0,075 \\
\hline Contato materno durante a gestação com óleos & $-0,036$ & 0,606 & 0,063 & 0,037 \\
\hline Contato materno durante a gestação com motores & $-0,091$ & 0,601 & 0,053 & $-0,277$ \\
\hline Contato materno durante a gestação com solventes & $-0,080$ & 0,530 & 0,176 & 0,005 \\
\hline $\begin{array}{l}\text { Contato materno durante a gestação com outras } \\
\text { substâncias químicas }\end{array}$ & $-0,002$ & 0,658 & 0,033 & $-0,057$ \\
\hline Contato materno durante a gestação com tintas & $-0,078$ & 0,447 & $-0,031$ & 0,122 \\
\hline Parto & $-0,053$ & 0,058 & 0,903 & 0,019 \\
\hline Anestesia & $-0,014$ & 0,072 & 0,898 & 0,083 \\
\hline Ultrassonografia & $-0,034$ & 0,042 & 0,126 & 0,492 \\
\hline Radiografia & $-0,068$ & $-0,121$ & 0,154 & 0,433 \\
\hline Uso de drogas materno durante a gestação & 0,101 & 0,074 & $-0,148$ & 0,554 \\
\hline Tingimento capilar materno & 0,124 & 0,085 & 0,033 & 0,474 \\
\hline Tingimento capilar paterno & 0,056 & 0,028 & $-0,053$ & 0,442 \\
\hline \% Variância explicada & 21,1 & 11,4 & 9,2 & 7,1 \\
\hline$\%$ Cumulativo & 21,1 & 32,5 & 41,7 & 48,8 \\
\hline
\end{tabular}

Fonte: Estudo Multi-institucional das Leucemias Infantis: Contribuição dos Marcadores Imuno-moleculares na Distinção de Diferentes Fatores Etiopatogênicos, 2007.

substâncias químicas (creosoto, asfalto ou betume, ácido clorídrico ou sulfúrico, colas e/ou outros), recebendo o nome de exposição ambiental e ocupacional materna na gestação.

Contabilizando 9,0\% da variância, o terceiro fator incluiu as variáveis tipo de parto (vaginal ou cesáreo) e realização de anestesia no parto, sendo denominado de assistência obstétrica.

O quarto fator incluído no modelo, que contribuiu com $7,0 \%$ da variância do modelo, é composto pela realização de exames radiológico e ultrassonográfico, uso de drogas pela mãe e tingimento capilar materno e paterno, sendo denominado como hábitos de vida e acesso a serviços de saúde.

O passo seguinte consistiu na realização da mesma análise separadamente para casos e controles. Nos casos de leucemia, o teste KMO observado correspondeu a 0,75, considerado adequado para análise fatorial, e o de Bartlett foi estatisticamente significativo ( $p$-valor $<0,0001$ ). Foram identificados três fatores que explicaram $50 \%$ da variância do conjunto de dados.

Assim como na análise do conjunto de dados, o primeiro fator foi denominado condições associadas ao hábito de fumar na gravidez, devido à sua composição similar anteriormente mencionada, sendo explicativo de $27 \%$ do total da variância. $\mathrm{O}$ segundo fator foi caracterizado pela exposição materna a poeiras, óleos, motores, solventes, tintas e outras substâncias químicas, explicando $14 \%$ da variância e denominado de condições associadas à exposição ambiental e ocupacional materna na gestação. 
O terceiro fator contabilizou $9,0 \%$ da variância e foi constituído pelo exame radiológico e ultrassonográfico, anestesia e tingimento capilar materno, tendo sido denominado como hábitos de vida e acesso a serviços de saúde.

$\mathrm{Na}$ análise fatorial realizada com os controles, o teste $\mathrm{KMO}$ observado correspondeu a 0,79 e o de Bartlett também foi estatisticamente significativo ( $p$-valor $<0,000)$. O modelo mais explicativo, contabilizando $55 \%$ da variância, foi formado por dois fatores a partir dos critérios mencionados anteriormente.

O primeiro, intitulado condições associadas ao hábito de fumar na gravidez, explicou $35 \%$ da variância do conjunto de dados e contemplou as seguintes variáveis originais: fumo durante a gestação; e consumo de fumo no 1ㅇ, 2 ㅇ e 3 을 trimestres.

O segundo fator, responsável por $20 \%$ da variância, foi composto pela exposição materna a: pesticidas, inseticidas e herbicidas; poeiras; óleos; motores; solventes; tintas; e outras substâncias químicas, recebendo o nome de exposição ambiental e ocupacional materna na gestação.

Com o intuito de determinar a magnitude de associação entre os fatores selecionados e a ocorrência de leucemias na infância, os escores obtidos com a análise fatorial para o conjunto de participantes (casos e controles) foram então empregados na modelagem com regressão logística. As razões de chance e os respectivos intervalos de confiança são apresentados no modelo inicial na Tabela 2. Por não terem sido encontradas diferenças importantes (superiores a $10 \%$ ), optou-se por utilizar as OR brutas em detrimento das ajustadas.

Os resultados da análise fatorial sugeriram que os fatores obtidos caracterizavam razoavelmente a amostra analisada (cerca de $50 \%$ da variância explicada). Em cada fator foi realizada a análise univariada, sendo incluídas no modelo multivariado as variáveis que apresentaram p-valor inferior a 0,20. Tanto no fator 1 (fumo em cada trimestre da gravidez) quanto no fator 3 (parto vaginal ou cesáreo e realização de anestesia), as variáveis eram bastante correlacionadas e adotou-se a maior magnitude da carga fatorial de cada uma daquelas variáveis como critério de seleção para a modelagem multivariada, sumarizando assim o fator nas quais se incluíam. Foram incluídos, então, o consumo de fumo no 2 o trimestre (carga fatorial $=0,982$ ) e parto (carga fatorial $=$ 0,903).

TABELA 2

Estimativas de razões de chance (OR) para portadores de leucemias agudas na infância no Brasil, segundo fatores obtidos pela análise fatorial.

\begin{tabular}{lc}
\hline Variáveis no modelo & $\begin{array}{c}\text { OR bruta } \\
\text { IC (95\%) }\end{array}$ \\
\hline Modelo inicial (1) & \\
$\quad$ Fator condições associadas ao hábito de fumar na gravidez & $0,97(0,81-1,16)$ \\
$\quad$ Fator exposição ambiental e ocupacional materna na gestação & $1,15(0,96-1,37)$ \\
$\quad$ Fator assistência obstétrica & $1,20(1,0-1,44)$ \\
$\quad$ Fator hábitos de vida e acesso a serviços de saúde & $1,08(0,90-1,30)$ \\
Modelo final (2) & \\
$\quad$ Fator exposição química materna durante a gestação & $1,35(1,16-1,58)$ \\
$\quad$ Fator hábitos de vida durante a gestação & $0,93(0,79-1,10)$ \\
$\quad$ Fator consumo de serviços de saúde durante a gestação & $1,27(1,08-1,48)$ \\
\hline
\end{tabular}

Fonte: Estudo Multi-institucional das Leucemias Infantis: Contribuição dos Marcadores Imuno-moleculares na Distinção de Diferentes Fatores Etiopatogênicos, 2007.

(1) Modelo inicial incluindo quatro fatores obtidos na análise fatorial inicial (62 variáveis),os quais contribuíram na explicação de $49 \%$ da variância dos dados.

(2) Modelo final incluindo três fatores obtidos na análise fatorial final (sete variáveis), os quais contribuíram na explicação de $52 \%$ da variância dos dados. 
Por serem de naturezas diferentes, todas as variáveis integrantes dos fatores 2 e 4 foram eleitas para análise univariada, totalizando 14 variáveis. Entre estas, apenas sete (consumo de fumo no $2 \mathrm{o}$ trimestre da gravidez; contato materno durante a gestação com pesticidas; solventes; tintas; radiografia; parto; e tingimento capilar materno durante a gestação) apresentaram p-valor abaixo de 0,20 na análise univariada, sendo incluídas no modelo multivariado.

Em seguida, foi realizada nova análise fatorial com as variáveis supracitadas e o modelo mais explicativo (52\% da variância) constituído por três fatores. O teste de KMO correspondeu a 0,55 e o teste de esfericidade de Bartlett foi estatisticamente significativo $(p<0,0001)$ (Tabela 3$)$.

A Tabela 3 apresenta as cargas fatoriais após a rotação varimax, sendo o primeiro fator constituído das variáveis contato materno durante a gestação com pesticidas, contato materno durante a gestação com solventes e contato materno durante a gestação com tintas, explicando 19,7\% da variância do modelo, sendo denominado exposição química materna durante a gestação.

O segundo fator, responsável por $17 \%$ da variância, composto pelo consumo de fumo no 2 o trimestre e tingimento capilar materno durante a gestação, foi designado hábitos de vida durante a gestação.
O terceiro fator contabilizou $15 \%$ da variância e contemplou as variáveis radiografia e parto, sendo intitulado consumo de serviços de saúde durante a gestação. Os componentes após a rotação varimax estão representados na Figura 1.

Após a realização da análise fatorial final (Figura 1), procedeu-se ao novo processo de modelagem com regressão logística, sendo as razões de chance entre os fatores identificados nesta etapa e a ocorrência de leucemias na infância apresentados na Tabela 2. Foram observadas associações estatisticamente significativas entre o desenvolvimento de leucemias na infância e as condições relacionadas ao fator denominado exposição química materna durante a gestação (OR=1,35, 95\% IC 1,16-1,58), bem como com as condições relacionadas ao fator consumo de serviços de saúde durante a gestação (OR=1,27, 95\% IC 1,08-1,48). Optou-se pelas razões de chance brutas, visto que não se observaram diferenças importantes em comparação às OR ajustadas.

\section{Discussão}

O modelo multivariado fatorial foi adotado, neste processo, por permitir a identificação de relações latentes entre um grande número de variáveis analisadas, possibilitando, assim, a síntese de informações em um

TABELA 3

Análise fatorial obtida por rotação varimax, modelo final

\begin{tabular}{lccc}
\hline Variáveis & $\begin{array}{c}\text { Fator 1 } \\
\text { Exposição química } \\
\text { materna durante a } \\
\text { gestação }\end{array}$ & $\begin{array}{c}\text { Fator 2 } \\
\text { Hábitos de } \\
\text { vida durante a } \\
\text { gestação }\end{array}$ & $\begin{array}{c}\text { Fonsumo de serviços } \\
\text { de saúde durante a } \\
\text { gestação }\end{array}$ \\
\hline Contato materno durante a gestação com pesticidas & 0,700 & $-0,093$ & $-0,059$ \\
Contato materno durante a gestação com tintas & 0,664 & $-0,064$ & $-0,064$ \\
Contato materno durante a gestação com solventes & 0,637 & 0,242 & 0,202 \\
Consumo de fumo no 2o trimestre & $-0,120$ & 0,756 & $-0,173$ \\
Tingimento capilar materno & 0,100 & 0,707 & 0,148 \\
Tipo de parto & 0,094 & $-0,118$ & 0,742 \\
Realização de radiografia & $-0,085$ & 0,100 & 0,662 \\
\hline \% Variância explicada & 19,7 & 16,7 & 15,5 \\
\% Cumulativo & 19,7 & 36,5 & 52,0 \\
\hline
\end{tabular}

Fonte: Estudo Multi-institucional das Leucemias Infantis: Contribuição dos Marcadores Imuno-moleculares na Distinção de Diferentes Fatores Etiopatogênicos, 2007. 
FIGURA 1

Representação espacial das relações entre fatores após rotação varimax, casos de leucemias pediátricas e controles, Brasil (1)

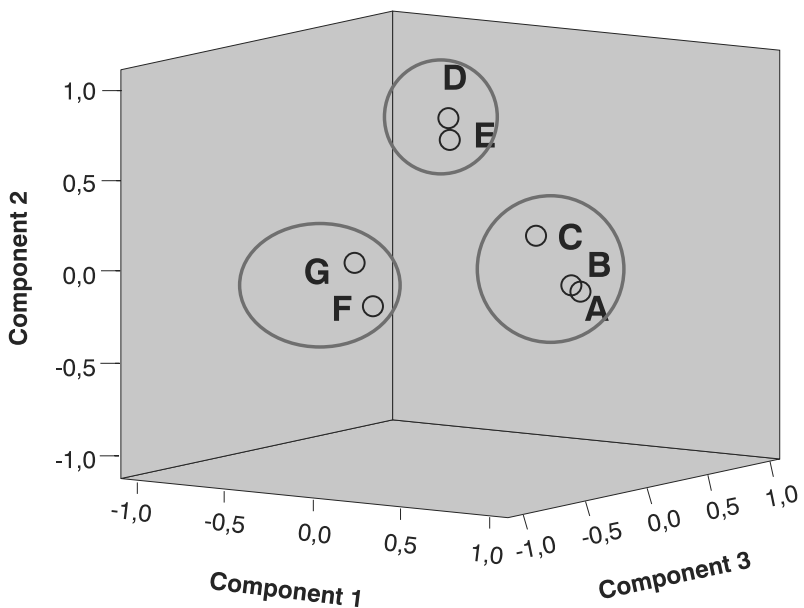

(1) Variáveis componentes do Fator 1: A (contato materno com pesticidas durante a gestação), B (contato materno com tintas durante a gestação) e $\mathrm{C}$ (contato materno com solventes durante a gestação). Variáveis componentes do Fator 2: $\mathrm{D}$ (consumo de fumo no $2^{\circ}$ trimestre) e $E$ (tingimento capilar materno durante a gestação). Variáveis componentes do Fator 3: $F$ (tipo de parto) $e$ $\mathrm{G}$ (exposição a radiação ionizante).

conjunto menor de fatores e a formulação de novas perguntas de pesquisa e/ou a confirmação de concepções causais preexistentes (HAIR et al., 1998; MINGOTI, 2005).

O emprego desta técnica permitiu a obtenção de um modelo explicativo de $49 \%$ da variância observada dos dados quando o método foi aplicado no conjunto de casos e controles. A análise das cargas fatoriais apontou o peso das condições relacionadas a tabagismo, exposições ambientais e ocupacionais, parto e puerpério e hábitos de vida e acesso a serviços de saúde.

Os resultados encontrados foram similares tanto na análise para o conjunto de participantes (casos e controles), quanto naquelas realizadas separadamente para cada um destes grupos, o que sugere uma consistência dos dados em relação à proveniência de ambos a partir de uma mesma população de estudo.

Após a identificação destes fatores, realizou-se modelagem multivariada, por meio da regressão logística não condicional, a fim de se confirmar sua contribuição na predição da ocorrência de leucemia na infância. Os resultados obtidos, entretanto, não revelaram uma contribuição substancial dos fatores neste sentido (Tabela 2, modelo inicial). Em processo de aperfeiçoamento do modelo inicial obtido, procedeu-se à sua reformulação, procurando incluir numa segunda etapa aquelas variáveis que isoladamente apresentavam maior significância estatística.

Com este subconjunto de variáveis, realizaram-se novas análises multivariadas (componentes principais, fatorial exploratória e regressão logística), as quais conduziram a identificação de fatores considerados mais adequados na predição das leucemias na infância.

Os resultados encontrados na presente investigação são apoiados por relatos de outros estudos, os quais mencionam as possíveis contribuições das exposições químicas na gravidez, hábitos de vida e consumo de serviços de saúde durante a gestação, todas estas de relevância para a saúde do recém-nascido, bem como no processo de carcinogênese (IARC, 1987; POMBO-DE-OLIVEIRA; KOIFMAN, 2006; SHU et al., 2002; INFANTE-RIVARD et al., 2003; KINLEN, 1995; MCNALLY et al., 2004; WEN et al., 2002; PANG et al., 2003; RUDANT et al., 2007). Neste sentido, os 
resultados apresentados nesta investigação reiteram a contribuição das exposições ambientais analisadas de forma conjunta, e não apenas individualizada, no desenvolvimento das leucemias na infância.

Entre os estudos já realizados, algumas investigações norte-americanas, asiáticas e europeias apontam a relevância das exposições ambientais na causalidade das leucemias na infância (PELISSARI et al., 2009; SWAMINATHAN et al., 2008; COEBERGH et al., 2006). Considerando a literatura sobre a exposição a substâncias químicas, o benzeno foi reconhecido como agente leucêmico (IARC, 1987), o que não ocorreu, entretanto, com grande parte dos agentes químicos. Neste sentido, ao não se identificar um potencial efeito carcinogênico, a exposição ocupacional e ambiental a estas substâncias químicas persiste.

Em relação à exposição às substâncias químicas contidas nas tinturas de cabelo, alguns estudos apontam o aumento no risco para câncer de mama em mulheres acima de 50 anos com estes antecedentes (SHORE et al., 1979). Entretanto, uma investigação de meta-análise realizada para determinar a associação entre este tipo de exposição em ambiente não ocupacional e o desenvolvimento de neoplasias em diferentes localizações anatômicas concluiu quanto à inexistência de elevação no risco de desenvolvimento de câncer de bexiga (dez estudos), câncer de mama (14 estudos) e neoplasias hematológicas (40 estudos) (TAKKOUCHE et al., 2005).

Em relação à exposição medicamentosa durante a gestação, um estudo caso-controle sugeriu que o uso de vitaminas e suplementos de ferro possa reduzir o risco de leucemias, enquanto o uso de anfetaminas e pílulas dietéticas poderia aumentar o risco de leucemia na infância (WEN et al., 2005). Outro estudo caso-controle acerca das exposições maternas durante a gestação e as leucemias na infância, realizado na população brasileira, indicou aumento de $40 \%$ no risco para o desenvolvimento das leucemias na infância com antecedentes de uso de dipirona no período gestacional (POMBO-DE-OLIVEIRA; KOIFMAN, 2006).
Este mesmo estudo apontou associação positiva entre o uso de pesticidas domésticos durante a gestação e discreta elevação estatisticamente significativa (18\%) no risco de leucemias na infância. Em apoio a estas observações, um estudo realizado na França comparou o uso de pesticidas entre mães de crianças portadoras de leucemias e linfomas e mães de controles, encontrando razão de chances mais elevada nas mães de casos do que nas dos controles, OR=2,2 (95\% IC 1,8-2,6). Mesmo tendo realizado a estratificação segundo subtipos leucêmicos, tipos de pesticidas e período de exposição, o estudo encontrou associações positivas na maior parte das comparações. Os autores concluem que o uso doméstico de pesticidas possa contribuir para a etiologia das leucemias e que a exposição pré-natal poderia constituir uma janela de vulnerabilidade fetal para o desenvolvimento de neoplasias hematológicas (RUDANT et al., 2007).

No que tange a outras exposições químicas, como é o caso daquelas decorrentes do tabagismo, ainda existem resultados controversos na literatura. Sabe-se, entretanto, que o fumo contém diversas substâncias carcinogênicas e alguns destes compostos foram encontrados na placenta, sangue fetal, urina de recém-natos e leite materno de fumantes, sugerindo o tabagismo como um comportamento de risco para o desenvolvimento de leucemias na infância (PANG et al., 2003).

Os resultados da presente investigação sugerem que as condições de atenção à saúde, incluindo a radiação ionizante, possam estar associadas à leucemogênese. $A$ exposição à radiação ionizante é um fator de risco reconhecidamente relacionado à ocorrência de leucemias, uma vez que estudos com sobreviventes das bombas atômicas no Japão apresentaram riscos elevados de leucemia atribuídos àquela exposição (WAKEFORD et al., 2003).

Quanto à radiação não ionizante, um estudo publicado por Wertheimer e Leeper (1979) sugeriu associação positiva entre a exposição a campos magnéticos de baixa frequência e leucemia em menores de 19 anos (WERTHEIMER; LEEPER, 1979). Pos- 
teriormente, diferentes investigações foram realizadas para analisar tal associação, com resultados controversos. Entretanto, é relativamente aceito na literatura que crianças expostas a campos magnéticos da ordem de 4,0 mG ou mais apresentam riscos elevados de desenvolverem leucemia (KHEIFETS et al., 2006). Desde 2002, o campo eletromagnético de baixa frequência é classificado, pela Agência Internacional de Pesquisa em Câncer da OMS (IARC/ WHO), como possível carcinógeno humano (KHEIFETS et al., 2006). No presente estudo, os resultados obtidos com a metodologia multivariada adotada (análise de componentes principais) não incluíram a exposição à radiação não ionizante durante a gravidez e no período pós-natal, em decorrência dos parâmetros de sua correlação estatística com as demais variáveis analisadas.

Devido à heterogeneidade no perfil genético, existem padrões distintos relacionados à capacidade individual de metabolização de carcinógenos e, consequentemente, à suscetibilidade individual de vir a desenvolver uma neoplasia (POMBO-DE-OLIVEIRA et al., 1986; PERERA, 1996; HUNGER et al., 1998; BHATIA et al., 1999; LABUDA et al., 2002; GREAVES, 2002). Dessa forma, além das exposições intrauterinas e após o nascimento, diversos fatores podem estar envolvidos no processo de leucemogênese na infância.

A multiplicidade de causas associadas ao desenvolvimento das leucemias pediátricas aponta para a importância de se utilizar um modelo que combine os vários fatores relacionados ao desenvolvimento da doença e possibilite evidenciar as diferenças entre estes. A utilização de medidas multidimensionais que possam resumir informações dentro de um número limitado de grupos é necessária para delinear panoramas de saúde para esta população. O método utilizado permitiu sintetizar um grande número de variáveis em um quantitativo reduzido de distintos grupos, consistentes com a literatura, possibilitando, assim, a validação dos resultados obtidos. Além disso, foi possível verificar como os padrões de exposição materna durante a gravidez, tais como as condições relacionadas à exposição a diversas substâncias químicas, aquelas associadas a hábitos de vida no período gestacional e à atenção obstétrica, se agrupam nesses indivíduos, podendo associar-se ao desenvolvimento de leucemias pediátricas.

Quanto às limitações do estudo, a ausência de trabalhos similares no Brasil e em outros países relativamente ao emprego da metodologia multivariada adotada dificultou a realização de comparações com os resultados encontrados na presente investigação. Em relação aos pontos fortes deste trabalho, destaca-se o fato de que foi analisado um banco de dados de abrangência nacional, tendo como objeto de estudo as leucemias na infância. Os resultados obtidos permitiram analisar conjuntamente diversos fatores associados à leucemogênese infantil, em vez da análise tradicional da busca de associações de natureza causal com variáveis isoladas. Adicionalmente, os resultados encontrados são sugestivos quanto à sua consistência e plausibilidade biológica, uma vez que os fatores obtidos por meio da metodologia adotada (análise de componentes principais e análise fatorial) incluem variáveis descritas na literatura como associadas à leucemogênese na infância.

Os estudos futuros deveriam privilegiar a incorporação deste tipo de análise, incluindo características genéticas das mães e crianças potencialmente indicativas dos mecanismos de suscetibilidade individual no processo de carcinogênese. A definição de padrões de exposição materna durante a gravidez fornece um conjunto de informações que permitem um melhor conhecimento desta população e entendimento do cenário da doença, além de propiciar subsídios para o planejamento de propostas de intervenção por meio de políticas públicas para a saúde infantil, em geral, e o controle do câncer, em particular.

\section{Conclusões}

A partir da análise de um banco nacional com dados clínicos e epidemiológicos no Brasil, foi possível observar que condições relacionadas à exposição materna a substâncias químicas durante a gravidez, a hábitos de vida neste período e à atenção 
obstétrica podem associar-se ao desenvolvimento de leucemia na infância. Estes fatores explicaram, respectivamente, $20 \%, 17 \%$ e $15 \%$ da variância dos dados, totalizando $52 \%$ da variância associada ao desfecho analisado.

Este estudo teve como escopo gerar hipóteses acerca dos padrões de exposições ambientais que podem estar associadas às leucemias na infância, podendo direcionar medidas de prevenção em saúde pública para crianças da população brasileira, sendo necessárias outras investigações realizadas com metodologias distintas.

\section{Agradecimentos}

Esta investigação foi parcialmente apoiada pela Swiss Bridge Foundation (grant 2301504) e pela Fogarty International Center, através do Irving J. Selikoff International Scholars Program, Mount Sinai School of Medicine (Award Number D43TW00640). O conteúdo do texto apresentado é de responsabilidade dos autores e não necessariamente representa o ponto de vista oficial da Fogarty International Center ou do National Institutes of Health, Estados Unidos. Maria do Socorro Pombo de Oliveira e Sergio Koifman têm suas atividades de pesquisa apoiadas pelo Conselho Nacional de Pesquisas e Desenvolvimento Científico (CNPq), respectivamente, \# 309091/2007 e 577598/2008-2.

\section{Pediatras do grupo de Estudo Colaborativo Brasileiro de Leucemia Aguda Infantil, que contribuíram como coautores do estudo}

Alejandro Arenciba - Hospital Santa Marcelina, São Paulo, SP

Atalla Mnayarji - Hospital Pedro Pedrossian, Campo Grande, Mato Grosso

Carmen M Mendonça - Serviço de Oncologia do Hospital Joana de Gusmão, Florianópolis, Santa Catarina

Claudia Julia - Hospital do Câncer 1-Inca; Hospital dos Servidores do Estado, e Hemorio, Rio de Janeiro

Cynthia Curvello Neves - Sociedade de Oncologia da Bahia, Salvador, Bahia
Ednalva Leite - Hospital Oswaldo Cruz, Ceon, Recife, Pernambuco

Eduardo Preto Serafini - Hospital Geral de Caxias do Sul, Rio Grande do Sul

Eloísa Cartaxo - Hospital Napoleão Laureano, João Pessoa, Paraíba

Eni Guimarães Carvalho - Hospital Martagão Gesteira, Salvador, Bahia

Fernando Augusto de Freitas - Centro Infantil de Investigações Hematológicas D. Boldrini, Campinas, São Paulo

Fernando Werneck - Departamento de Pediatria, Hospital dos Servidores do Estado do Rio de Janeiro

Flávia Nogueira Serafim Araújo - Sociedade de Oncologia da Bahia, Salvador, Bahia

Flávia Pimenta - Hospital Napoleão Laureano, João Pessoa, Paraíba

Gilberto Ramos - Departamento de Pediatria, Faculdade de Medicina, UFMG, Belo Horizonte, Minas Gerais

Isis Q. Magalhães - Hospital de Apoio Brasília, Unidade de Onco-Hematologia Pediátrica, Brasília, DF

Jane Dobbin - Hospital do Câncer 1-Inca; Hospital dos Servidores do Estado, e Hemorio, Rio de Janeiro

Jose Andréa Yunes - Centro Infantil de Investigações Hematológicas D. Boldrini, Campinas, São Paulo

Jose Carlos Cordoba - Hospital de Apoio Brasília, Unidade de Onco-Hematologia Pediátrica, Brasília, DF

Jozina Aquino - Sociedade de Oncologia da Bahia, Salvador, Bahia

Jozina Maria de Andrade Agareno - Sociedade de Oncologia da Bahia, Salvador, Bahia

Lilian Burlemaqui - Sociedade de Oncologia da Bahia, Salvador, Bahia

Lilian Maria Burlacchini de Carvalho - Hospital Martagão Gesteira, Salvador, Bahia

Maria Célia Moraes Guerra - Instituto de Pediatria e Puericultura Martagão Gesteira, UFRJ, Rio de Janeiro

Maria Lucia Lee

Maurício de Souza Meira - Hospital Martagão Gesteira, Salvador, Bahia

Maurício Dumond - Sociedade de Oncologia da Bahia, Salvador, Bahia

Nilma Pimentel de Brito - Hospital Martagão Gesteira, Salvador, Bahia

Núbia Mendonça - Sociedade de Oncologia da Bahia, Salvador, Bahia 
Paulo Ivo C. Araújo - Instituto de Pediatria e Puericultura Martagão Gesteira, UFRJ, Rio de Janeiro Reinaldo Del Belo - Centro de Pesquisa e Serviço de Hematologia, Instituto Nacional de Câncer, Rio de Janeiro

Renato Melaragno - Hospital Santa Marcelina, São Paulo, SP

Ricardo Bigni - Centro de Pesquisa e Serviço de Hematologia, Instituto Nacional de Câncer, Rio de Janeiro

Rosania Maria Basegio - Hospital Pedro Pedrossian, Campo Grande, Mato Grosso

\section{Referências}

BELSON, M.; KINGSLEY, B.; HOLMES, A. Risk factors for acute leukemia in children: a review. Environmental Health Perspectives, v. 115, n. 1, p. 138-145, Jan. 2007.

BHATIA, S.; ROSS, J. A.; GREAVES, M. F.; ROBINSON, L. L. Epidemiology and etiology. In: PUI, C. H. (Ed.). Childohood leukemias. Cambridge: Cambridge University Press, 1999, p. 38-49.

BRAGA, P. E.; LATORRE, M. R. D. O.; CURADO, M. P. Câncer na infância: análise comparativa da incidência, mortalidade e sobrevida em Goiânia (Brasil) e outros países. Cad. Saúde Pública, v. 18, n. 1, p. 33-44, 2002.

COEBERGH, J. W.; REEDIJK, A. M.; VRIES, E. de; MARTOS, C.; JAKAB, Z.; STELIAROVA FOUCHER, E.; KAMPS, W. A. Leukemia incidence and survival in children and adolescents in Europe during 1978-1997. Reported from the Automated Childhood Cancer Information System Project. Eur. J. Cancer, v. 42, n. 13, p. 2019-2036, 2006.

DICKINSON, H. O. The causes of childhood leukaemia: delayed exposure to infection may trigger leukaemia after prenatal damage to DNA. BMJ, v. 330, p. 1279-1280, 2005.

GREAVES, M. Molecular genetics, natural history and the demise of childhood leukemia. Eur. J. Cancer, v. 35, n. 14, p. 1941-1953, 1999.

GREAVES, M. F. Childhood Leukemia. BMJ, v. 324, p. 283-287, 2002.
Silvia Maia - Hospital do Câncer 1-Inca; Hospital dos Servidores do Estado, e Hemorio, Rio de Janeiro

Tereza Cristina Cardoso

Terezinha J. M. Salles - Hospital Oswaldo Cruz, Ceon, Recife, Pernambuco

Virginia M. Coser - Departamento de Hematologia, Universidade de Santa Maria, Rio Grande do Sul

HAMERSCHLAK, N. Leucemia: fatores prognósticos e genética. J. Pediatr., v. 84, n. 4, suppl. p. S52-57, 2008.

HAIR, J. F.; TATHAM, R. L.; ANDERSON, R. E.; BLACK, W. Análise fatorial. In: HAIR, J. F.; TATHAM, R. L.; ANDERSON, R. E.; BLACK, W. (Orgs.). Análise multivariada dos dados. 5 a ed. São Paulo: Artmed Editora, 1998, p. 89-124.

HOSMER, D. M.; LEMESHOW, S. Applied logistic regression. New York: Joh Wiley and Sons, 1989.

HUNGER, S. P. et al. Oncogenesis in utero: fetal death due to acute myelogenous leukaemia with an MLL translocation. Br. J. Haematol., v. 103, n. 2, p. 539-542, 1998.

IARC. Monographs on the evaluation of carcinogenic risks to humans. Suplement 7: overall evaluations of carcinogenicity - an updating of IARC Monographs, volumes 1 to 42,1987 . Disponível em: <http:// monographs.iarc.fr/ENG/Monographs/ suppl7/Suppl7-24.pdf $>$. Acesso em: 07 fev. 2010.

INFANTE-RIVARD, C.; DEADMAN, J. E. Maternal occupational exposure to extremely low frequency magnetic fields during pregnancy and childhood leukemia. Epidemiology, v. 14, n. 4, p. 437-441, 2003.

KHEIFETS, L.; AFIFI, A. A.; SHIMKHADA, R. Public health impact of extremely lowfrequency electromagnetic fields. Env. Health Perspect., v. 114 , n. 10 , p. $1532-$ 1537, 2006. 
KINLEN, L. J. Epidemiological evidence for an infective basis in childohood leukaemia. Br. J. Cancer, v. 71, n. 1, p. 1-5, 1995.

LABUDA, D. et al. Parental genotypes in the risk of a complex disease. Am J. Hum. Genet., v. 71, n. 1, p. 193-197, 2002.

LINET, M. S.; RIES, L. A.; SMITH, M. A.; TARONE, R. E.; DEVESA, S. S. Cancer surveillance series: recent trends in childhood cancer incidence and mortality in the United States. J. Natl. Cancer Inst., v. 91, p. 1051-1058, 1999.

MCNALLY, R. J. Q.; EDEN, T. O. B. An infectious aetiology for childhood leukaemia: a review of the evidence. Br. J. Haematology, v. 127, p. 243-246, 2004.

MARCHIONI, D. M. L.; LATORRE, M. R. D. O.; ELUF-NETO, J.; WÜNSCHFILHO, V.; FISBERG, R. M. Identification of dietary patterns using factor analysis in an epidemiological study in São Paulo. São Paulo Med. J., v. 123, n. 3, p. 124-127, 2005.

MINGOTI, S. A. Análise fatorial. In: MINGOTI, S. A. (Org.). Análise de dados através de métodos de estatística multivariada: uma abordagem aplicada. Belo Horizonte: UFMG, 2005, p. 99-141.

PANG, D.; MCNALLY, R.; BIRCH, J. M. Parental smoking and childhood cancer: results from the United Kingdom Childhood Cancer Study. British J. of Cancer, v. 88, n. 3, p. 373-381, 2003.

PELISSARI, D. M.; BARBIERI, F. E.; FILHO, V. W. Magnetic fields and acute lymphoblastic leukemia in children: a systematic review of case-control studies. Cad. Saúde Pública, v. 25, supl. 3, p. S441-S452, 2009.

PERERA, F. P. Molecular epidemiology: insights into cancer susceptibility, risk assessment and prevention. J. Nat. Cancer. Inst., v. 88, n. 8, p. 496-509, 1996.

POMBO-DE-OLIVEIRA, M. S. et al. Lymphoblastic leukemia in siamese twins: evidence for identity. Lancet, v. 2, n. 8513, p. 969-970, 1986.

Programa de Hematologia

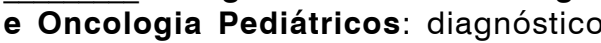

imunomolecular. 2008. Disponível em: $<$ www.imunomolecular.redecancer.org.br>. Acesso em: 07 ago. 2010.

POMBO-DE-OLIVEIRA, M. S.; KOIFMAN, $S$. Infant acute leukemia and maternal exposures during pregnancy. Cancer Epidemiol. Biomarkers Prev., v. 15, n. 12, p. 2336-2341, 2006.

PUI, C. H.; SANDLUND, J. T.; PEI, D.; RIVERA, G. K.; HOWARD, S. C.; RIBEIRO, R. C. et al. Results of therapy for acute lymphoblastic leukemia in black and white children. Jama, v. 290, p. 2001-2007, 2003.

REIS, R. S.; SANTOS, M. O.; THULER, L. C. S. Incidência de tumores pediátricos no Brasil. Rev. Bras. de Cancerologia, v. 53, n. 1, p. 5-15, 2007.

SHORE, R. E.; PASTERNACK, B. S.; THIESSEN, E. U.; SADOW, M.; FORBES, R.; ALBERT, R. E. A case-control study of hair dye use and breast cancer. J. Natl. Cancer Inst., v. 62, n. 2, p. 277-83, Feb. 1979.

SHU, X. O.; POTTER, J. D.; LINET, M. S.; SEVERSON, R. K.; HAN, D.; KERSEY, J. H.; JOSEPH, P. N.; TRIGG, M. E.; ROBINSON, L. L. Diagnostic X-rays and ultrasound exposure and risk of childhood acute lymphoblastic leukemia by immunophenotype. Cancer Epidemiol. Biomarkers Prev., v. 11, p. 177-185, 2002.

SWAMINATHAN, R.; RAMA, R.; SHANTA, V. Childhood cancers in Chennai, India, 19902001: incidence and survival. Int. J. Cancer, v. 122, p. 2607-1611, 2008.

RUDANT, J.; MENEGAUX, F.; LEVERGER, G. et al. Household exposure to pesticides and risk of childhood hematopoietic malignancies: the scale study (SFCE). Environ Health Perspect., v. 115, n. 12, p. 1787-1793, 2007.

TAKKOUCHE, B.; ETMINAN, M.; MONTESMARTINEZ, A. Personal use of hair dyes and risk of cancer: a meta-analysis. Jama, v. 293, n. 20, p. 2516-2525, 2005.

WAKEFORD, R.; LITTLE, M. P. Risk coefficients for childhood cancer after intrauterine irradiation: a review. Int. J. Radiat. Biol., v. 79, n. 5, p. 293-309, 2003. 
WERTHEIMER, N.; LEEPER, E. Electrical wiring configuration and childhood cancer. Am. J. Epidemiol., v. 109, p. 273-284,1979.

WEN, W.; SHU, X. O.; POTTER, J. D.; SEVERSON, R. K.; BUCKLEY, J. D.;
REAMAN, G. H.; ROBISON, L. L. Parental medication use and risk of childhood acute lymphoblastic leukemia. Cancer, v. 95, p. 1786-1794, 2002.

\section{Resumen}

Exposiciones ambientales y leucemias en la infancia en Brasil: un análisis exploratorio de su asociación

El presente estudio tiene como objetivo explorar las relaciones no aparentes que diversos factores relacionados con exposiciones ambientales, y características individuales existentes en nuestro medio, pueden llegar a tener en el proceso de desarrollo de la leucemia en la infancia. A partir de un banco de datos clínicos y epidemiológicos, obtenido con un estudio de control de casos de base hospitalaria sobre factores de riesgo para leucemias en la infancia, se realizó un análisis multivariado exploratorio, mediante el empleo de un análisis de componentes principales y análisis factorial. Esta investigación es parte de un estudio multicéntrico nacional que incluyó 292 casos de leucemias en niños con edades comprendidas entre 0 y 12 años y 541 controles de la misma franja etaria, hospitalizados por causas no neoplásicas en hospitales generales, cercanos a los centros de origen de los casos. La información sobre las exposiciones ambientales seleccionadas se obtuvo mediante entrevistas realizadas con madres de casos y controles a través de un cuestionario padronizado. El modelo con mayor poder explicativo de la variancia observada en los datos analizados fue del orden de un $52 \%$, presentando tres factores considerados como más adecuados en la predicción de la leucemogénesis en la infancia, cada uno incluyendo variables con cargas factoriales mayores que 0,6: factor "condiciones relacionadas con las exposiciones químicas en la gestación", el cual explicó un $20 \%$ de la variancia final e incluyó las variables exposición a pesticidas, exposición a solventes y exposición a tintas en el embarazo; factor "hábitos de vida durante la gestación", habiendo explicado un $17 \%$ de la variancia e incluyendo exposición a tintes de pelo y cosméticos para el alisamiento; el factor "consumo de servicios de salud durante la gestación", explicando un $15 \%$ de la variancia e incluyendo las variables tipo de parto (cesárea o parto normal) y uso de radiografías en la gestación. En el análisis de regresión logística, fue encontrada una asociación estadísticamente significativa entre el desarrollo de leucemias en la infancia y antecedentes de exposición química materna durante la gestación $(O R=1,36 ; 95 \% I C=1,16-1,59)$ y consumo de servicios de salud durante la gestación $(O R=1,27 ; 95 \% I C=1,08-1,49)$. Los resultados encontrados son sugestivos en lo que se refiere a la contribución conjunta de las exposiciones ambientales, y no sólo individualizadas, en el desarrollo de las leucemias en la infancia. Siendo apoyados por evidencias en la literatura especializada de que el proceso de carcinogénesis, en general, y/o de la leucemogénesis en particular, resultan de efectos de múltiples mutaciones relacionadas a exposiciones ambientales conjuntas.

Palabras-clave: Leucemia. Infancia. Exposiciones ambientales. Análisis factorial. Regresión logística.

\section{Abstract}

Environmental exposure and childhood leukemia in Brazil: an exploratory analysis of their association

This study aims to explore the unapparent relations that several factors related to environmental exposure and individual characteristics existing in our environment may have with the process of 
developing childhood leukemia. From a database obtained from a clinical and epidemiological hospital-based, case-control study on risk factors for childhood leukemia, an exploratory multivariate analysis was performed using principal component analysis and factor analysis. This research is part of a national multicenter study that included 292 cases of leukemia in children aged 0 to 12 years and 541 controls of the same age, hospitalized for non-neoplastic causes in general hospitals near the centers the cases originated in. Information on selected environmental exposure was obtained in interviews with the mothers of both cases and controls by means of a standardized questionnaire. The model with the greatest explanatory power for the variance observed in the data analyzed was of approximately $52 \%$. Three factors were considered most appropriate for predicting leukemogenesis in childhood, each including variables with factor loadings greater than 0.6 : factor "conditions related to chemical exposures during pregnancy", which explained $20 \%$ of the final variance and included the variables pesticide exposure, exposure to solvents and paint exposure in pregnancy; factor "lifestyle habits during pregnancy" explained $17 \%$ of the variance and included exposure to hair dyes and cosmetics for hair straightening; and factor "use of health services during pregnancy", which explained $15 \%$ of the variance and included the variables type of delivery (vaginal or caesarean) and use of radiography in pregnancy. Logistic regression analysis revealed a statistically significant association between the development of leukemia in childhood and maternal history of chemical exposure during pregnancy $(\mathrm{OR}=1.36,95 \% \mathrm{Cl}=1.16-1.59)$ and use of health services during pregnancy $(\mathrm{OR}=1.27,95 \% \mathrm{Cl}=1.08-1.49)$. The results indicate the joint contribution of not just individual but environmental exposure in the development of leukemia in childhood, and are supported by evidence in the literature that the process of carcinogenesis in general and of leukemogenesis in particular, result from effects of multiple mutations related to joint environmental exposure.

Keywords: Leukemia. Childhood. Environmental exposure. Factor analysis. Logistic regression.

Recebido para publicação em 16/02/2012

Aceito para publicação em 22/06/2012 